\title{
A New Method for a Remote Control of a Robotic Manipulator
}

$\underline{\text { https://doi.org/10.3991/ijoe.v17i09.23913 }}$

\author{
Seifedine Kadry ${ }^{1}\left({ }^{\varpi}\right)$, G. Alferov ${ }^{2}$, V. Korolev, D. Shymanchuk ${ }^{2}$ \\ ${ }^{1}$ Noroff University College, Kristiansand, Norway \\ ${ }^{2}$ Saint-Petersburg State University, Saint-Petersburg, Russia \\ skadry@gmail.com
}

\begin{abstract}
A new method for a remote control of a space robot designed to perform operations in a complex external environment. It's dealing with objects that can move freely in space or limited by holonomic bonds that limit their possible movements. This is a typical situation when performing assembly operations in discovering space. The method is based on the use of an important feature that characterizes each operation performed on the interaction of the working tool of the robot with the external objects in the environment.
\end{abstract}

Keywords - copy control, remote control, adaptive control, sustainability of control processes

\section{$1 \quad$ Introduction}

A method for controlling remote operations that operate in a complex external environment when control signals is delayed was described. The method is based on the use of a special operation performed when the robot working tool interacts with environmental objects. The proposed approach provides for the separation of the remotecontrol process into two stages.

The first stage, carried out on the ground control center, is the stage of training the robot to the required action. At the first stage, the control is not carried out by the robot itself, but a very good model of it. The model must function in an environment that serves as a model of the real external environment of the robot. In this environment, a person must perform the required operation using a robot model. Since there are no delays in the transmission of control signals from the handle to the robot model on the ground control center, the required operation can be successfully carried out. Other methods of performing the operation in a model environment, for example, with the help of a master glove, are also acceptable.

The second stage involves the execution of this action by a real space robot. It can be argued that in the case of using these laws as software for the control system of a real robot with a sufficiently high quality of their tracking by this system at the second stage of control of a space robot, there is a very high probability of the robot performing the required operation in a nondeterministic external environment. 
In the first stage by means of appropriate sensors mounted on a robot model, in the process of performing the required operations are formed the laws of change in time series data characterizing the interaction strength of the working tool model with the objects of the external environment of the robot, as well as their mutual position.

It can be argued that in the case of using these laws as software for the control system of a real robot with a sufficiently high quality of their tracking by this system at the second stage of control of a space robot, there is a very high probability of the robot performing the required operation in a nondeterministic external environment. This class is a set of arbitrarily spaced solids that can be either freely movable in space or have holonomic bonds $[1,25]$ limiting their possible displacements.

The above statement is based on the following consideration. For each type of operation on interaction of the working tool (fight) with a subject of the environment there is a certain invariant-the passport of its performance [2-6]. It includes the trajectory of changes in time position relative to the grip of the so-called characteristic points belonging to the objects of the environment. In the case of force interaction of the grip with objects, the law of change of force and moment of interaction of the grip with these objects, measured by the wrist force-moment sensor, is added to these trajectories "in time".

For successful performance of the robot working tool operations with the subject, it is necessary that in the process of implementation of the operation, the position of the object relative to the working tool, as well as the magnitude of the force of the interaction of the tool with the subject were identical to the position and forces of their models in the learning process, i.e. that the passport of the operation obtained by the models in the learning process coincided with the passport of the implemented operation.

Thus, for the implementation of the proposed approach, it is necessary to equip the robot model with a set of sensors, which provides the possibility of forming "passport" trajectories used as software at the training stage. To track these trajectories, the control system of a real space robot must use information similar to that used to construct passport trajectories. This requires equipping a real space robot with the same sensor software that was used on its model, installed in a similar way.

In the case of a sufficiently accurate tracking of the aforementioned software working tool trajectories of the real robot, and the target operation object in a nondeterministic external environment is performed regardless of the possible inaccuracies of the model of the external environment in which the ongoing process of learning, mainly due to the difference of actual positions of objects in the base coordinate system of the robot body from their model positions. To improve the accuracy of tracking software trajectories, instead of the traditional control "deviation" it is advisable to use more advanced control methods, for example, combined control, when the mismatch signal is formed as the difference between the program current values and supplemented by a signal of change in the value of control, which took place in the learning process

The proposed method of remote control is obviously a method of off-line remote control, which largely removes the time delay restrictions in the transmission of control signals and receiving feedback signals. This is achieved by the fact that the feed- 
back signals on the position and strength of the interaction of the robot with the external environment are closed not through a person, as in traditional bilateral control, but through the control system of the space robot, located near the Executive organs of the space robot, which eliminates the delay.

The described approach is more effective in the implementation of the remote, stationary or quasi-stationary conditions, when objects in the scene do not move too fast. However, it is still functional, in the case of free-moving objects, the environment, and, in the case when the motion of the objects is limited by the relationship. For example, it is an object of this type can be a case with ports on which you want to insert the discs that move along guides. It is possible that the recording medium on the surface of the profile that needs to be sanded down with a special tool to press on the ground with enough force and effect. It is also possible to make the connection between the two parts, one of which has a hole in it, and the other pin inserted in the hole, there is also an option for the environment.

The activities described above, and similar ones can be, in principle, be used for the creation of an appropriate interpreter, a problem-oriented language used for the implementation of the supervised control of a space robot [25].

When working in the room, and the advantage of the proposed approach is most fully revealed, as well as the area in which the robot is operating in an area where it's hard to objects of irregular shape, are quite spread out. Such a surface can be found on the surface of some asteroids, the Moon and Mars.

\section{Substantiation of the efficiency of the approach.}

To clarify the efficiency of the proposed approach to the organization of remote control of a space robot, it is necessary to conduct a detailed analysis of the dynamic properties of the remote-control system of the robot implemented based on this approach. As a result of the analysis conditions of stability of functioning of system of remote control of the robot must be revealed. Only if they are satisfied, the system can be recognized as workable. Since the remote-control system includes a ground and space part, for its performance, first, must be satisfied the requirements of sustainability for bilateral controlled from the master arm of the ground robot model, and secondly, to be achieved stability of the local control system of a real space robot which must track the programmed trajectory generated from the ground control center, real robot.

As for the dynamic analysis [3] of the functioning of a bilaterally controlled model of a space robot from the control arm, it has already been carried out [4] and its results, including the conditions for achieving stability of the control, are given in [8-10, 15-18]. This article presents a dynamic analysis of the process of functioning of the local control system of the space robot while tracking the program trajectories formed in the process of training at the ground control center.

The dynamic description of the behavior of a manipulative space robot functioning in weightlessness has the form:

$$
A \ddot{q}+B \dot{q}+C q=H U+Q,
$$




$$
\begin{gathered}
A=\frac{\partial^{2} T}{\partial(\dot{q})^{2}}, \\
B=\frac{\partial A}{\partial t}-\frac{1}{2}\left[\begin{array}{c}
\partial(\dot{q})^{T} A \\
\partial q
\end{array}\right]+k_{F}+k_{V}, \\
C q=-\frac{\partial \Pi}{\partial q}, \quad C=\left[\begin{array}{ll}
0 & 0 \\
0 & C_{e}
\end{array}\right], \quad H=\left[\begin{array}{l}
E \\
0
\end{array}\right],
\end{gathered}
$$

where $q=(g, e), \dot{q}=(\dot{g}, \dot{e})-(n+m)$ - dimensional vectors of generalized coordinates of the robot and its velocity vectors a managed g coordinate construction accordingly $g$ and elastic $e$ coordinate construction accordingly, $e-m$ - dimensional vector of deformations of elastic elements of design manipulation robot, A and C $(n+m) \times(n+m)$ symmetric positive-definite matrix of inertia and paleopedology stiffness matrix, $C_{e}-(m \times m)$ symmetric positive-definite matrix of stiffness of the robot, $Q-(n+m)$ - dimensional vector of generalized reactions of connections (forces of interaction of the working tool with objects of the environment having sclerotomies communications), $H-(n+m) \times n$ - block matrix, $k_{F}-(n+m) \times$ $(n+m)$ - symmetric positive definite matrix of coefficients of friction, $E-n \times n$ unit matrix, $U-n$ - dimensional vector of control.

- Generalized coordinates of the manipulator mechanism included in the vector $q$ may be "related". This is the case if the grip of the manipulator moves an object whose possible movements are limited by bonds, most likely sclerotomies. The object, rigidly grasped by the grip, is one with the grip, so the connections of the object act as the ties of the grip of the manipulator. The equations of relations have the form

$$
M(X)=M(f(q))=0,
$$

where $M(X)-r$ - dimensional continuously differentiable vector function $r \leq 6$.

Here $X=\left(Y_{1}, Y_{2}, Y_{3}, \theta_{1}, \theta_{2}, \theta_{3}\right)$ - vector of position and orientation of the working tool (grip) of the space manipulator attached to the last link of the manipulator with the help of an "elastic" element, which is a wrist force-moment sensor that measures the amount of force applied to the grip by the objects of the external environment moved by it. $Y_{1}, Y_{2}, Y_{3}$ - coordinates of the pole (the origin of the coordinate system associated with the working tool), $\theta_{1}, \theta_{2}, \theta_{3}$ - Euler angles of rotation of the coordinate system of gripper.

The differential form (2) has the form:

$$
\frac{\partial M(X)}{\partial X} \dot{X}=0,
$$

and in a more convenient normalized form

$$
p \dot{X}=p S \dot{q}=0,
$$


where $p=R^{-1} \partial M / \partial X-r \times 6$ is the normalized linkage matrix, $R=$ $\operatorname{diag}\left\{R_{1}, R_{2}, \ldots, R_{6}\right\}$ - diagonal $r \times r$ - matrix, $R_{j}$ - Euclidean norm of the $j$ - th row of the matrix: $\partial M / \partial q, S=\frac{\partial X}{\partial q}-6 \times(n+m)$ - Jacobi matrix.

For small increments $\Delta X, \Delta q$ the equation connecting them has a form similar to (4):

$$
p \Delta X=p S \Delta q=0 .
$$

The vector $\Delta X$ can be represented as the sum of two terms:

$$
\Delta X=\Delta X_{g}+\Delta X_{e},
$$

where $\Delta X_{g}-n$ - dimensional increment vector generated by the increment $\Delta g$ of the vector of controlled generalized coordinates, and $\Delta X_{e}-m$ - dimensional increment vector generated by the increment of the vector $\Delta e$ of elastic deformations of the robot structure.

Instead of the vector $\dot{X}^{i}$ in the equations of relations, you can use the vector

$$
\dot{x}=(\omega, v)=\beta \dot{X},
$$

where $\beta$ is an orthogonal $6 \times 6$ - matrix, which is a function of the vector $q$ of angular $\omega$ and linear $v$ velocities of the Tong of the space manipulator, in the coordinate system that is associated with the Tong. Instead of the vector $\Delta X$ we apply the vector of small increments:

$$
\Delta x=\beta \Delta X .
$$

Then, considering (7), (8), the expression (6) can be reduced to the following normalized form of representation of the coupling equations:

$$
p J \Delta X=p \Delta X_{g}+p \Delta X_{e}=p J_{g} \Delta g+p J_{e} e=0,
$$

where $J=\left[J_{g} J_{e}\right]-6 \times(n+m)$ - the Jacobian matrix of the manipulator, $J_{g}$ and $J_{e}-6 \times n$ and $6 \times m$ blocks.

Due to the smallness of the deformation $e$ in (9) $\Delta e$ is replaced by $e . J_{g}=\left[J_{g}^{v} \mid J_{g}^{\omega}\right]$ $-6 \times n$ Jacobian matrix for controlled generalized velocities, $J_{g}^{v}$ and $J_{g}^{\omega}-3 \times n$ blocks connecting vectors of linear $v$ and angular $\omega$ velocities.

Obviously, the vector $Q$ of generalized bond reactions, i.e. the vector of the force of interaction of the grip with objects of the external environment, referred to the vector $q$ of generalized coordinates of the robot, has the following representation: $Q=\left(Q_{g}, Q_{e}\right)=J^{T} p^{T} \lambda$, where $\lambda-r$ - dimensional vector - Lagrange multiplier, $Q_{g}$ and $Q_{e}$ - vectors of generalized reactions, referred to the vectors of controlled $g$ and elastic $e$ coordinates of the structure, respectively. They are presented as

$$
Q_{g}=J_{g}^{T} p^{T} \lambda \text { и } Q_{e}=J_{e}^{T} p^{T} \lambda .
$$


- To analyze the control process, first of all it is necessary to obtain a dynamic description of the closed control system of a real space robot. The desired description is obtained using the system (1) when replacing the vector $U$ in it with its representation.

$$
U=\frac{1}{n} \sum_{i=1}^{n}\left(\frac{\partial X^{i}}{\partial g}\right)^{T} K_{p}\left(X_{d}^{i}-X^{i}\right)-K_{Q}\left(Q_{g}^{d}-Q_{g}\right),
$$

where $Q_{g}^{d}$ and $Q_{g}$ - are vectors of the desired and current values of the forces of interaction of the grip with the object of the external environment, referred to the vector of controlled generalized coordinates of the robot. Unfortunately, the current value of the vector $Q_{g}$, included in this expression for $U$ is very inconvenient for its formation, since the exact value of this value requires measurement with the help of appropriate sensors not only the variables $g$ and $e$, which are components of the generalized coordinates $q=(g, e)$, but also their derivatives.

Therefore, in practice, a value close to the required value is used as $Q_{g}$, however, it is exactly equal to it only when $\dot{q}=q=0$. These values can be obtained using a subsystem consisting of the last $\mathrm{m}$ equations of the system (1) at $\dot{q}=q=0$, which leads this system to the form $C_{e} e=Q_{e}=J_{e}^{T} p^{T} \lambda$ and, since according to (10) $Q_{g}=$ $J_{g}^{T} p^{T} \lambda$, the sought $Q_{g}=J_{g}^{T} C_{r} J_{e} e$, where $C_{r}=\left[J_{e} C_{e}^{-1} J_{e}^{T}\right]^{-1}$ matrix stiffness of the wrist force-moment sensor. The current value of $Q_{g}$ is formed by a force-moment sensor, which in the process of implementing the control measures the value of $e$.

As for the other quantities included in the control law $U$, namely the quantities $X^{i}, i=1,2,3, \ldots, n$, they are formed in the above manner by laser rangefinders or by CCD cameras.

Finally, the formation of the current values of the matrices $\left(\frac{\partial X^{i}}{\partial g}\right)^{T}, i=$ $1,2,3, \ldots, n$, included in the expression for the control vector $U$, is also quite simple. A more detailed representation of these matrices can be obtained by using equality

$$
x^{i}=x_{0}+\alpha X^{i},
$$

where $x^{i}$ and $x_{0}$ are vectors representing the base coordinate system of robot $i$-th characteristic point of the subject stationary in the external environment and the end (beginning) of the moving coordinate system associated with the end of the last link of the manipulator (mounting location to the link in the body from the force-torque sensor), $\alpha$ - matrix guides of the cosines of the rotation coordinate system of the link relative to the base system.

Differentiating the right and left parts of this equality, we get

$$
\frac{\partial X^{i}}{\partial q} \dot{g}=\alpha^{T}\left(\dot{x}^{i}-\dot{x}_{0}\right)+\dot{\alpha}^{T}\left(x^{i}-x_{0}\right) .
$$

Due to the stationarity of the external environment, the position vectors of the characteristic points $x^{i}$ do not depend on time, i.e. $\dot{x}^{i}=0$. Then, taking into account 
that $\dot{\alpha}^{T}=-\alpha^{T} \omega$ and that $\alpha^{T} \dot{x}_{0}=v=J_{g}^{v}$, and $\omega=J_{g}^{\omega} \dot{g}$, where $v$ - vectors of linear velocity of the pole (origin) of the last link of the manipulator, $\omega$ - vector of angular velocity of the last link in the coordinate system of this link, finally, taking into account that $\alpha^{T}\left(x^{i}-x_{0}\right)=X^{i}$, the equality (11) can be

$$
\frac{\partial X^{i}}{\partial q} \dot{g}=J_{g}^{v} \cdot \dot{g}+X^{i} J_{g}^{\omega} \dot{g} .
$$

Therefore

$$
\frac{\partial X^{i}}{\partial q}=J_{g}^{v}+X^{i} J_{g}^{\omega}
$$

Further dynamic analysis requires modernization of the classical description of the behavior dynamics of the mechanism having sclerotomies connections. Instead of describing the dynamics in the form of two systems of equations, one of which is a system of Lagrange differential equations of the II kind, the second is a system (9) consisting of $\mathrm{r}$ equations of sclerotomies connections, we will use an upgraded system.

It is obtained from the source (1) by substituting in it $r$-dimensional Lagrange multiplier $\lambda$, which is the vector of generalized reactions of the relations $Q=J^{T} \lambda$ value $c p J \Delta q$, in which $c \rightarrow \infty$, and $c p J \Delta q \rightarrow 0$ each $j$-th component which is proportional to the distance $p_{j} J \Delta q$ point defined by the vector $q$ to the $j$-th hypersurface, represented by the equation $p_{j} J \Delta q=0$, where $p_{j}-j$ - th row of the matrix $p$. It is the $j$ - th equation included in the system of coupling equations (9).

The scalar matrix $\mathrm{c} \rightarrow \infty$ is used as the proportionality matrix. The validity of the described substitution is shown in [15].

In accordance with the above, assuming that $q-q_{0}=\Delta q$ - is a small increment, and $q_{0}-$ is the value of $q$, satisfying the coupling equation (4), we have

$$
\lambda=-A p J\left(q-q_{0}\right)=-c p J \Delta q, A \rightarrow \infty
$$

Thus, the new representation of the system of equations of dynamics of the manipulator model (1) will take the form

$$
A \ddot{q}+B \dot{q}+C q=H U-J^{T} p^{T} c p J \Delta q
$$

and will only include differential equations.

After the transition in this system to a new variable $\Delta=q-q_{0}, q=(g, e), \Delta_{r}=$ $g-g_{0}, \Delta_{e}=e-e_{0}$, where $q_{0}, e_{0}, g_{0}$ correspond to the equilibrium state of the model, replace $U$ with its representation (8). As a result, we obtain the following system of equations by additionally carrying out the linearization of the system about $\dot{q}=$ $0, q=q_{0}$,

$$
\ddot{\Delta}+\dot{\Delta}+C \Delta=0
$$

where 


$$
A=\left[\begin{array}{ll}
A_{n n} & A_{n m} \\
A_{m n} & A_{m m}
\end{array}\right], B=\left[\begin{array}{cc}
B_{n n} & 0 \\
0 & B_{m m}
\end{array}\right]=k_{F}+k_{V} \text { и } C=\left[\begin{array}{cc}
f_{n n} & f_{n m} \\
f_{m n} & f_{m m}
\end{array}\right]
$$

$-(n+m) \times(n+m)$ matrices of inertia, representations of dissipative forces, and representations of forces dependent on the vector of generalized coordinates.

$$
\begin{aligned}
& f_{n n}=\left(J_{g}\right)^{T} p^{T} c p J_{g}+\frac{1}{n} \sum_{i=1}^{n}\left(\frac{\partial X^{i}}{\partial \Delta_{g}}\right)^{T} K_{p}\left(\frac{\partial X^{i}}{\partial \Delta_{g}}\right) \\
& f_{n m}=k_{R} J_{g}^{T} C_{r} J_{e}+J_{g}^{T} p^{T} c p J_{e}, \\
& f_{m n}=J_{e}^{T} p^{T} c p J_{g}, f_{m m}=c_{e}+J_{e}^{T} p^{T} c p J_{e} .(13)
\end{aligned}
$$

To clarify the stability conditions of the obtained system (11), we will carry out the following transformations. First, we present it in the normal resolved form with respect to the first derivative. Secondly, we show that the system transformed in this way, in the case of the following design features of the manipulator, will include two subsystems of differential equations. One of them is singularly perturbed, i.e. has a small parameter at derivatives, the second subsystem is undisturbed. The abovementioned feature of the manipulator design, which generates such a structure, is that the mass-inertial characteristics of the links must be much greater than the same characteristics of the working tool attached to the last link of the manipulator through the elastic structure of the wrist force-moment sensor.

Thirdly, let us find out whether the above system satisfies the conditions of Tikhonov's theorem [11]. If they are satisfied, it becomes possible to successfully conduct a dynamic analysis, for example, to clarify the stability, with a much simpler system of differential equations, namely, with the so-called generating system, into which the original system of differential equations turns if you put a small parameter for derivatives in a singularly perturbed subsystem equal to zero. Fourth, we will perform the required analysis of the generating subsystem in order to determine its stability. A positive result makes it possible to reasonably extend it to the original system.

In order to bring the system (12) to the normal, resolved with respect to the first derivative form, taking into account that $\Delta=\left(\Delta_{r}, \Delta_{e}\right)$, we introduce new variables $\Delta_{r 1}=\dot{\Delta}_{r}, \Delta_{e 1}=\dot{\Delta}_{e}$ and get

$$
\begin{gathered}
\dot{\bar{\Delta}}_{r 1}=-\Pi_{\text {or }} \bar{\Delta}, \\
\dot{\bar{\Delta}}_{e 1}=-\Pi_{o e} \bar{\Delta}, \\
\bar{\Delta}_{r}=\left(\Delta_{r}, \Delta_{r 1}\right), \bar{\Delta}_{e}=\left(\Delta_{e}, \Delta_{e 1}\right), \bar{\Delta}=\left(\Delta_{r}, \bar{\Delta}_{e}\right), \\
\Pi_{0 r}=\left[\begin{array}{ccccc}
0 & E_{r} & 0 & 0 \\
\Phi_{r n} & T_{0 r} & \Phi_{r m} & 0
\end{array}\right] \text { и } \Pi_{0 e}=\left[\begin{array}{cccc}
0 & 0 & 0 & E_{e} \\
\Phi_{e n} & 0 & \Phi_{e m} & T_{0 e}
\end{array}\right],
\end{gathered}
$$

where $\Pi_{0 r}$ and $\Pi_{0 e}-2 n \times 2(n+m)$ and $2 m \times 2(n+m)$ - matrices;

$$
T_{0 r}=a_{n} \cdot B, T_{0 e}=a_{m} \cdot B,
$$




$$
\begin{gathered}
\Phi_{0 r}=\left[\begin{array}{ll}
\Phi_{r n} & \Phi_{r m}
\end{array}\right], \Phi_{0 e}=\left[\begin{array}{ll}
\Phi_{e n} & \Phi_{e m}
\end{array}\right], \\
\Phi_{r n}=a_{n}\left(f_{\bullet n}\right), \Phi_{r m}=a_{n \bullet}\left(f_{\bullet m}\right), \\
\Phi_{e n}=a_{m \bullet}\left(f_{\bullet n}\right), \Phi_{e m}=a_{n \bullet}\left(f_{\bullet n}\right), \\
\left(A_{0}\right)^{-1}=\left[\begin{array}{ll}
a_{n n} & a_{n m} \\
a_{m n} & a_{m m}
\end{array}\right]=\left[\begin{array}{c}
a_{n \bullet} \\
a_{m \bullet}
\end{array}\right] ; \\
C=\left[\begin{array}{l}
f_{n \bullet} \\
f_{m \bullet}
\end{array}\right]=\left[\begin{array}{ll}
f_{n n} & f_{n m} \\
f_{m n} & f_{m m}
\end{array}\right]=\left[\begin{array}{ll}
f_{\bullet n} & f_{\bullet m}
\end{array}\right],
\end{gathered}
$$

$E_{r}$ and $E_{e}-(n \times n)-$ и $(m \times m)-$ unit matrix blocks.

Blocks $a_{n n}, a_{n m}, a_{m n}, a_{m m}$ matrix $\left(A_{0}\right)^{-1}$ in (17) the dimensions $n \times n, n \times m$, $m \times n, m \times m$ are represented through blocks $A_{n n}, A_{n m}, A_{m n}, A_{m m}$ matrix $A_{0}$ using the Frobenius formula

$$
\begin{gathered}
a_{n n}=A_{n n}^{-1}+A_{n n}^{-1} A_{n m} a_{m m} A_{m n} A_{n n}^{-1}, \\
a_{n m}=a_{m n}^{T}=A_{n n}^{-1} A_{n m} a_{m m}, a_{m m}=\left(A_{m m}-A_{m n} A_{n n}^{-1} A_{n m}\right)^{-1} .
\end{gathered}
$$

Let's introduce a constant parameter:

$$
\mu^{2}=\Pi_{r} \Pi_{e}^{-1} \text {. }
$$

Rules $\Pi_{r}, \Pi_{e}$ legitimate to replace the norms of the blocks $\Phi_{o r}, \Phi_{o e}$, since the elements of unit blocks $E_{r}, E_{e}$, and the elements of block matrices $T_{o e}, T_{o r}$ the coefficients of viscous friction are much smaller than the elements of the matrices $\Phi_{o r}$. The latter are matrices of parameters that determine the values of potential and elastic forces of the manipulator. These parameters are much more than one, as well as the coefficients of viscous friction. Therefore, the expression (19) for $\mu^{2}$ can be replaced:

$$
\mu^{2}=\Phi_{o r} \Phi_{o e}^{-1}=a_{n} \cdot a_{m} \bullet^{-1} \leq\left(a_{n n}+a_{n m}\right) a_{m m}{ }^{-1},
$$

where $a_{n n}, a_{m m}, a_{m \bullet}, a_{n \bullet}$ - the octahedral norms of matrices $a_{n n}, a_{m m}, a_{m \bullet}, a_{n \bullet}$. With (18) for $a_{n m}$ and $a_{n n}$ let us be convinced of the fairness of the inequality:

$$
a_{n m} \leq A_{n n}^{-1} A_{n m} a_{m m}<a_{n n} A_{n m} a_{m m} .
$$

Replacing in (21) the value $a_{n m}$ with the right part of the inequality (18), we give (19) to view the form

$$
\mu^{2}<a_{n n}\left(a_{m m}{ }^{-1}+A_{n m}\right) .
$$

And since $a_{m m}{ }^{-1}<A_{m m}$, which follows from (18) for $a_{m m}$, finally have

$$
\mu^{2}<a_{n n}\left(A_{m m}+A_{n m}\right),
$$

where $a_{n n}=A_{n n}^{-1}+A_{n n}^{-1} A_{n m} a_{m m} A_{m n} A_{n n}^{-1}<\operatorname{Cond}\left(A_{n n}\right) A_{n n}{ }^{-1}(E+\delta)$

$$
\delta<\operatorname{Cond}\left(A_{n n}\right) A_{n n}{ }^{-1} A_{n m} A_{m m} A_{m n} \leq E
$$


The analysis showed that the design features of the considered class of manipulators, as well as the smallness of the mass and moment of inertia of the working tool fixed at the end of the manipulator, compared with the inertia and mass of its other movable elements - the manipulator links, lead to the following characteristic relationship between the matrix blocks.

The value of the block $A_{n n}$ is mainly determined by the moments of inertia of the manipulator links. It is much larger than the size of the blocks $A_{n m}=A_{m n}^{T}, A_{m m}$, which are determined by the moment of inertia and the mass of the working tool at the end of the manipulator, i.e. the following inequality takes place:

$$
A_{n n}>>A_{m n}, A_{n n}>>A_{n m} \text { и } A_{n n}>>A_{m m} \text {. }
$$

Therefore, as can be seen from (20), the parameter $\mu^{2}$ will be very small if the block $A_{n n}$ of the matrix $A$ is also sufficiently well-conditioned, i. e. $\operatorname{Cond}\left(A_{n n}\right)$ will not be too large.

To further transform systems (14), (15) using a small parameter $\mu$ we introduce instead $\Delta_{e 1}$ variable $\tilde{\Delta}_{e 1}=\mu \dot{\Delta}_{e}$ and multiply by $\mu^{2}=\Phi_{o r} \Phi_{o e}^{r-1}$ the right and left parts of the equality (15). We also replace matrix $\Pi_{o r}$ with a representation $\Phi_{o r}$ using the expression (16). As a result, we obtain the following record of the systems of equations (14), (15):

$$
\begin{aligned}
\mu \dot{\Delta}_{e} & =\tilde{\Delta}_{e 1}, \\
\mu \dot{\tilde{\Delta}}_{e 1} & =-\mu T_{o e} \tilde{\Delta}_{e 1}-\Phi_{o r} \Phi_{o e}^{r}{ }^{-1} \Phi_{o r} \Delta, \\
\dot{\Delta}_{r} & =\Delta_{r 1}, \\
\dot{\Delta}_{r 1} & =-T_{o r} \Delta_{r 1}-\Phi_{o r} \Delta+k \Delta_{r} .
\end{aligned}
$$

It includes an undisturbed subsystem (24) and a singularly perturbed subsystem (23), since its left parts have a small scalar multiplier $\mu$ for the derivatives of the variables $\Delta_{e}, \Delta_{e 1}$. At the same time, the multiplier $\Phi_{o r} \Phi_{o e}{ }^{-1} \Phi_{o e}$ for the variable $\Delta$ in the right part of this subsystem is commensurable with the matrix coefficient $\Phi_{o r}$ for the same variable in the subsystem (22), since it is the product of the norm $\Phi_{o r}$ of the matrix $\Phi_{o r}$ on the normalized matrix $\Phi_{o e}{ }^{-1} \Phi_{o e}$.

If this subsystem (23), (24) satisfies Tikhonov's theorem [11], this means, firstly, that, at $\mu=0$ a singularly perturbed system linking the variables $\Delta_{e}$ and $\Delta_{r}$, turns into an algebraic system that has an isolated root $\Delta_{e 0}=\left(\Delta_{e}, \Delta_{e 1}\right)=\varphi\left(\Delta_{r}\right)$. Secondly, when replacing the variable $\Delta_{r}$ included in this singular subsystem with some parameter, for example zero, the latter turns into a system of differential equations that is stable in the neighborhood of $\Delta_{e 0}$, then for the dynamic analysis of the original system (23) and (24) it is possible to use a simpler, than the original, so-called generating subsystem in the first approximation. It is obtained from the non-perturbed subsystem of (24) when you replace a variable included in it $\Delta_{e}$ isolated root $\Delta_{e 0}=$ $\varphi\left(\Delta_{r}\right)$

Taking into account (16), (17) for $\Phi_{0 e}, C$ and $a_{m \bullet}$, as well (13) for $f_{n n}, f_{n m}$ and (18) for $a_{m n}$ and $a_{m m}$, have 


$$
\Delta_{e 10}=0, \Phi_{o e} \Delta=a_{m \bullet} f_{01} \Delta=a_{m m}\left(A_{m n} A_{n n}^{-1} f_{n} \bullet+f_{m \bullet}\right) \Delta=0 .
$$

The norm of the matrix coefficient $A_{m n} A_{n n}^{-1}$ at $f_{n}$. cannot exceed $A_{m n} A_{n n}^{-1}<$ $a_{n n} A_{n m}$, as follows from (21). This value is less than $\mu^{2}$, as can be seen from the expression (22). Then it is logical to neglect in (25) the term $a_{m m} A_{m n} A_{n n}^{-1}$, and the subsystem (25), taking into account the no degeneration of $a_{m m}$ will turn into a subsystem

$$
f_{m} \cdot \Delta=0 .
$$

Using (17) for $f_{m \bullet}=\left[\begin{array}{ll}f_{m n} & f_{m m}\end{array}\right]$ and (13) for $f_{m n}, f_{m m}$, write the system (26) as

$$
C_{e} \Delta_{e}+J_{e}^{T} p^{T} c p J_{g} \Delta_{g}+J_{e}^{T} p^{T} c p J_{e} \Delta_{e}=0
$$

Hence

$$
\Delta_{e}=\left(C_{e}+J_{e}^{T} p^{T} c p J_{g}\right)^{-1} J_{e}^{T} p^{T} c p J_{g} \Delta_{g} .
$$

To form a generating system in the system (24) it is necessary to replace the vector $\Delta_{e}$, which is a block of the vector $\Delta$, with its representation (28) through the vector $\Delta_{r}$.

In section 3 of the publication [25] it was shown that the vector $\Phi_{0 r} \Delta$, included in (24) and represented in accordance with (16), (17) as $\Phi_{0 r} \Delta=a_{n} .\left[\begin{array}{ll}f_{\bullet n} & f_{\bullet m}\end{array}\right] \Delta$, at $\mu \rightarrow$ 0 is defined with great precision as

$$
\Phi_{0 r} \Delta=a_{n n}\left(f_{n n} \Delta_{r}+f_{n m} \Delta_{e}\right) .
$$

After replacing $f_{n n}$ and $f_{n m}$ with their expressions (13), replacing $\Delta_{e}$ with its representation (28) and corresponding transformations, we have

$$
\begin{gathered}
f_{0 r}=\frac{1}{n} \sum_{i=1}^{n}\left(\frac{\partial X^{i}}{\partial \Delta_{g}}\right)^{T} K_{p}\left(\frac{\partial X^{i}}{\partial \Delta_{g}}\right)+\left(k_{R}+E\right) J_{g}^{T} C_{e} J_{g}-\left(k_{R}+E\right) J_{g}^{T} C_{e}\left(C_{e}+\right. \\
\left.p^{T} c p\right)^{-1} C_{e} J_{g}
\end{gathered}
$$

If we put the matrix coefficients $k$ and $k_{R}$ as scalar positive matrices and take into account that $C_{e}-$ is a positive-definite matrix, then $f_{o r}$. is a symmetric positivedefinite matrix. Indeed, all three terms forming $f_{o r}$, are symmetric matrices by virtue of their structure, whence follows the symmetry of $f_{o r}$. In addition, the first two terms are positive-definite matrices, and the third term is negative - definite. However, this term in sum with the second cannot be a negative-definite matrix, which follows from the obvious inequality $C_{e} \geq C_{e}\left(C_{e}+p^{T} c p\right)^{-1} C_{e}$. Therefore $f_{o r}$ is symmetric and positively defined.

Replacement in (24) member $\Phi_{0 r} \Delta_{r}$ its expression (29) gives the following representation of the generating subsystem of equations for the manipulator model in the form of a second-order equation:

$a_{n n}^{-1} \ddot{\Delta}_{r}+\mathrm{A}_{n n} \ddot{\Delta_{r}}+f_{0 r} \Delta_{r}=0$, 
where $\mathrm{A}_{n n}$ - is a diagonal positive-definite matrix composed of the first $n$ rows of the matrix $k_{F}+k_{V}$. In accordance with the above statement, the obtained system (31) allows to analyze the dynamics of the initial system (14) in the first approximation, i.e. to be a generating system if the attached system is stable.

As for the generating system, its stability, and hence the stability of the analyzed initial system will take place since the matrix coefficient $B_{n n}$ as the coefficients $a_{n n}^{-1}$ and $f_{0 r}$ - symmetric positive-definite matrices. The attached system is obtained from a singularly perturbed subsystem (23) in which the variable $\Delta_{r}$, which is a block of the vector $\Delta=\left(\Delta_{r}, \Delta_{e}\right)$, is assigned a parameter, such as zero: $\Delta_{r}=0$.

Replace in (23) the matrix multiplier $\Phi_{o e}$ with its expression (16), and the expressions of matrix $a_{m}$. and matrix $C$ - with their representations (17), followed by the formula (13) for $f_{m n}^{I}, f_{m n}^{I}$ and formulas (17) for $a_{m n}$ and $a_{m m}$. These actions transform (23) into the following form of recording the attached subsystem of equations:

$$
\mu \dot{\Delta}_{e 1}=-\mu T_{0} \Delta_{e 1}-\Phi_{o r} \Phi_{o e}{ }^{-1} a_{m m}\left[f_{m m}+A_{m n} A_{n n}^{-1} f_{n m}\right] \Delta_{e} .
$$

Member $A_{m n} A_{n n}^{-1}<a_{n n} A_{m n}<\mu^{2}$, as follows from (21). Therefore, in (32) the summands $A_{m n} A_{n n}^{-1} f_{n m}$ it is logical to neglect at $\mu \rightarrow 0$. Then the subsystem (32) in the form of second-order equations will take the form:

$$
\mu a_{m m} \ddot{\Delta_{e}}+\mu B_{m m} \dot{\Delta}_{e}+\Phi_{o r} \Phi_{o e}^{-1} f_{m m} \Delta_{e}=0,
$$

where $B_{m m}$ - a diagonal positive-definite $m \times m$ - matrix composed of the last $\mathrm{m}$ rows of the matrix $B=k_{F}+k_{V}$.

Since all matrix coefficients under variables $\ddot{\Delta}_{e}, \dot{\Delta}_{e}, \Delta_{e}$-positive definite symmetric matrices, including $f_{m m}$, what follows from (15) for $f_{m m}$, that (33) describes a steady process. The obtained result testifies to satisfaction of the conditions of the Tikhonov theorem [11] for the system of equations (12) the dynamics model of robot that gives you the right to use to analyze the dynamics model of the robot instead of the system of equations (12) generates the system of equations (31) since, as shown above, he describes a steady process.

\section{Conclusion}

In this article the method of remote control of the space robot intended for performance in the nondeterministic external environment of various operations with subjects as freely moved in space, and having holonomic communications is offered and proved. The latter is typical when performing Assembly operations most in demand at the present time in space exploration.

The method is based on the use of important features that characterize each operation to be performed on the interaction of the working tool of the robot with objects in the external environment. It includes the law of change in time of a vector of force and the moment of interaction, and the law of change of a vector of position of object in coordinate system of the working tool. The type of operation does not depend on the position and orientation of the object with which the robot working tool interacts in the basic coordinate system. The structure of the positional power of the robot 
controller, the synthesized control laws, the design of the manipulator, which provide the stability of process control, which guarantees the performance of the proposed method.

\section{$4 \quad$ Acknowledgment}

This work is dedicated to the blessed memory of F.M. Kulakov.

\section{$5 \quad$ References}

[1] Seifedine Kadry, Gennady Alferov, Viktor Fedorov, D-Star Algorithm Modification, International Journal of Online and Biomedical Engineering (iJOE), Vol 16, No 08 (2020). https://doi.org/10.3991/ijoe.v16i08.14243

[2] Seifedine Kadry, Bilal Ghazal, Design and Assessment of Using Smartphone Application in the Classroom to Improve Students' Learning, International Journal of Engineering Pedagogy (iJEP), Vol 9, No 2 (2019). https://doi.org/10.3991/ijep.v9i2.9764

[3] F.M. Kulakov, Seifedine Kadry, G.V. Alferov, P.A. Efimova, Remote Control of Space Robots Change-Adaptive in its External Environment, International Journal of Online and Biomedical Engineering (iJOE), Vol 15, No 07 (2019). https://doi.org/10.3991/ijoe. $\underline{\mathrm{v} 15 \mathrm{i} 07.10219}$

[4] Kulakov F., Alferov G., Sokolov B., Gorovenko P., Sharlay A. Dynamic analysis of space robot remote control system, AIP Conference Proceedings. St. Petersburg. 2018. V. 1959. P. 080014. https://doi.org/10.1063/1.5034731

[5] Kulakov F., Sokolov B., Shalyto A., Alferov G. Robot Master Slave and Supervisory Control with Large Time Delays of Control Signals and Feedback, (2016) Applied Mathematical Sciences. V. 10 (33-36). P. 1783-1796. https://doi.org/10.12988/ams.2016.6380

[6] Kulakov F., Alferov G., Sokolov B., Sharlay A. Bilateral Remote Control over Space Manipulators, (2018) AIP Conference Proceedings. 2040, P. 150015. https://doi.org/10.1063/1 .5079218

[7] Kulakov F., Kadry S., Alferov G., Efimova P., Remote Control of Space Robots ChangeAdaptive in its External Environment, International Journal of Online and Biomedical Engineering, 15 (7), 2019, pp. 84-98. https://doi.org/10.3991/ijoe.v15i07.10219

[8] Kulakov F.M., Methods of Supervisory Remote Control over Space Robots // Journal of Computer and Systems Sciences International, 57(5), pp. 822-839. https://doi.org/10.1134/ $\underline{\mathrm{s} 1064230718050088}$

[9] Kulakov F.M, Methods of Supervisory remote control of space robots, Proceedings of the Russian Academy of Sciences. Theory and control systems. 2018. No. 5, pp. 161-181.

[10] Ivanov G., Alferov G., Efimova P. Integrability of nonsmooth one-variable functions, 2017 Constructive Nonsmooth Analysis and Related Topics (Dedicated to the Memory of V.F. Demyanov), CNSA 2017 - Proceedings, 7973965. https://doi.org/10.1109/cnsa. 2017.7973965

[11] Tikhonov A. N. Systems of differential equations containing small parameters at derivatives. Mat. sat. 1952. Vol. 31. No. 3. C. $575-586$.

[12] Alferov G.V., Ivanov G.G., Efimova P.A. The structural study of limited invariant sets of relay stabilized system (Book Chapter) (2017) Mechanical Systems: Research, Applications and Technology, pp.101-164. 
[13] Ivanov G., Alferov G., Gorovenko P., Sharlay A. Estimation of periodic solutions number of first-order differential equations (2018) AIP Conference Proceedings, 1959, 080006. https://doi.org/10.1063/1.5034723

[14] Alferov G.V., Ivanov G. G., Efimova P.A., Sharlay A.S. Stability of linear systems with multitask right-hand member (Book Chapter) (2018) Stochastic Methods for Estimation and Problem Solving in Engineering, pp.74-112. https://doi.org/10.4018/978-1-52255045-7.ch004

[15] Alferov G., Ivanov G., Efimova P., Sharlay A. Study on the structure of limit invariant sets of stationary control systems with nonlinearity of hysteresis type (2017) AIP Conference Proceedings, 1863, 080003. https://doi.org/10.1063/1.4992264

[16] Ivanov G., Alferov G., Sharlay A., Efimova P. Conditions of asymptotic stability for linear homogeneous switched systems, (2017) AIP Conference Proceedings, 1863, P. 080002. https://doi.org/10.1063/1.4992263

[17] Kadry, S., Alferov, G., Kondratyuk, A., Kurochkin, V., Zhao, S. Modeling the motion of a space manipulation robot using position control, (2019) AIP Conference Proceedings, 2116, P. 080005. https://doi.org/10.1063/1.5114065

[18] Kadry, S., Alferov, G., Ivanov, G., Sharlay, A. About stability of selector linear differential inclusions (2018) AIP Conference Proceedings, 2040, P. 150013. https://doi.org/10. $1063 / 1.5079216$

[19] Kadry, S., Alferov, G., Ivanov, G., Sharlay, A. Stabilization of the program motion of control object with elastically connected elements (2018) AIP Conference Proceedings, 2040, P. 150014. https://doi.org/10.1063/1.5079217

[20] Kadry, S., Alferov, G., Ivanov, G., Korolev, V., Selitskaya, E. A new method to study the periodic solutions of the ordinary differential equations using functional analysis (2019) Mathematics, 7(8), 677. https://doi.org/10.3390/math7080677

[21] Kadry, S., Alferov, G., Ivanov, G., Sharlay, A. Almost Periodic Solutions of First-Order Ordinary Differential Equations (2018) Mathematics, V.6, №9, P.171. https://doi.org/ $\underline{10.3390 / \text { math6090171 }}$

[22] Kulakov F.M., Remote control of space robots, Izvestia RAS. Theory and control systems. 2016. No. 4. Pp. 141-192.

[23] Kulakov F.M., Methods of Supervisory remote control of space robots, Proceedings of the Russian Academy of Sciences. Theory and control systems. 2018. No. 5. Pp. 161-181.

[24] Polyakhova E.N., Korolev V.S., Problems of spacecraft control by solar sail, International Conference "Stability and Oscillations of Nonlinear Control Systems" (Pyatnitskiy's Conference 2016). https://doi.org/10.1109/stab.2016.7541214

[25] Kulakov F. M., Alferov G. V., Efimova P. A. Copying control of remote robots with delay in signal transmission. Bulletin of Perm state University. Mathematics. Mechanics. Informatics. 2019. 3 (46). Pp. 47-55. https://doi.org/10.17072/1993-0550-2019-3-47-55

\section{Authors}

Seifedine Kadry is with Noroff University College, Kristiansand, Norway.

G. Alferov is with Saint-Petersburg State University, Faculty of Applied Mathematics and Control Processes, Saint-Petersburg, Russia.

D. Shymanchuk is with Saint-Petersburg State University, Faculty of Applied Mathematics and Control Processes, Saint-Petersburg, Russia.

Article submitted 2021-05-11. Resubmitted 2021-06-20. Final acceptance 2021-06-21. Final version published as submitted by the authors. 